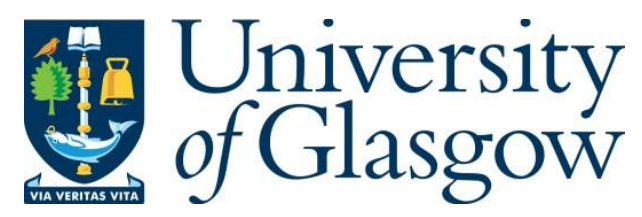

Busin, V., Burgess, S. and Shu, W. (2018) A hybrid paper-based microfluidic platform toward veterinary P-ELISA. Sensors and Actuators B: Chemical, 273, pp. 536-542.

There may be differences between this version and the published version. You are advised to consult the publisher's version if you wish to cite from it.

http://eprints.gla.ac.uk/164823/

Deposited on: 11 July 2018

Enlighten - Research publications by members of the University of Glasgow http://eprints.gla.ac.uk 


\title{
A hybrid paper-based microfluidic platform toward veterinary P-ELISA
}

\author{
Valentin Busin ${ }^{a, b}$ Stewart Burgess ${ }^{a}$ Wenmiao Shu ${ }^{b, c}$ \\ a Moredun Research Institute, Pentlands Science Park, Bush Loan, Edinburgh EH26 OPZ, \\ United Kingdom \\ b School of Engineering and Physical Sciences, Heriot-Watt University, Edinburgh EH14 4AS, \\ United Kingdom \\ c Department of Biomedical Engineering, University of Strathclyde, Glasgow, G4 ONW, United \\ Kingdom
}

\section{Abstract}

This study describes a novel fabrication method to produce a hybrid paper-based microfluidic platform, termed Multi-Pad Paper Plate $\left(\mathrm{MP}^{3}\right)$ for transfer and optimisation of enzyme-linked immunoassays (ELISA) on paper (P-ELISA). The new fabrication technique is based on a combination of laser micromachining of paper and packaging through thermal lamination. This method is simple, rapid and of high-resolution and can produce robust, versatile and low-cost devices, compatible with a standard 96-well microplate format. The MP ${ }^{3}$ was used to transfer a sandwich ELISA for detection of bovine haptoglobin ( $\mathrm{Hp})$, a marker of inflammation in animals allowing optimisation of the assay in the new format. Using the $\mathrm{MP}^{3}$, a standard curve was generated and the limit of detection achieved was $0.73 \mu \mathrm{g} / \mathrm{ml}$. The optimised protocol was also applied to the detection of $\mathrm{Hp}$ in bovine serum, demonstrating the possibility of using this platform with biological samples. The new platform allowed for a reduction of $93 \%$ in time and of $88 \%$ in cost for performing the assay and represents a valid alternative to other commonly used device fabrication methods, especially in the context of lowresource settings. It has the potential to translate similar assays into P-ELISA and offers a starting point for achieving animal-side testing.

\section{Introduction}

One of the most promising technologies that have recently been applied within point-of-care (POC) diagnostics is microfluidics [1]. At present, two main types of microfluidic systems are used in the diagnostic field: micro total analysis systems ( $\mu$ TAS) and microfluidic paper-based analytical devices ( $\mu$ PADs). The latter, in particular, represent an appealing application for low resource settings, due to their low-cost and ease of fabrication. Paper is cheap, easy to source, biodegradable and simple to chemically modify [2]. Furthermore, POC devices made from paper have the considerable advantage of not requiring external power sources, whilst fabrication techniques and machinery for production are usually cheaper than those required for other materials [3]. Paper represents an excellent medium for diagnostic testing, because of its high surface to volume ratio, which allows reagents to be concentrated and also enables more rapid reaction times [4]. 
Within the field of $\mu P A D s$, an interesting application has recently been demonstrated in the translation of ELISA onto paper (P-ELISA) [[4], [5], [6], [7], [8]]. P-ELISA combines the sensitivity and specificity of ELISA with the intrinsic low cost and ease-of-use of paper-based platforms [9]. There are increasing numbers of studies reporting the use of paper ELISA as a valid alternative to the conventional format $[6,7,10]$. The most commonly used methods for fabrication of these platforms are photolithography [10] and wax printing techniques $[6,8,11]$. The main drawbacks are the numerous steps involved for production of the final device [4], the cost of the use of photoresist [12], poor channel resolution [12] and the lack of resistance to sustain high temperature. To overcome these limitations a new concept of low-cost device has also been introduced, called paper hybrid microfluidic platforms [13]. A combination of paper and other materials, like PMMA [14], PDMS [15], cotton [16] or glass [17] are combined to fully exploit the properties of each material [13], reducing the limitations and drawbacks of the previous techniques. However, the current fabrication methods are still limited by the speed of prototype devices and high cost for scalable manufacturing.

Within veterinary diagnostics, the use of haptoglobin $(\mathrm{Hp})$ as a marker of disease status has recently attracted considerable attention [[18], [19], [20], [21], [22]]. Hp is classified as a serum biomarker, one of a number of acute phase proteins which increases rapidly following infection or inflammation, providing a quantification of tissue damage in a diseased animal [23]. $\mathrm{Hp}$ is normally present in the serum of healthy cattle in concentrations $<0.02 \mathrm{mg} / \mathrm{ml}$ [24], but a level rise above $25 \%$ of the normal circulating level is usually indicative of a diseased status [25]. Measurement of $\mathrm{Hp}$ in bovine serum is currently available with laboratory-based assays, either through a colorimetric reaction based on peroxidase activity of the haptoglobin-haemoglobin complex or with a commercially available ELISA kit [26]. Furthermore, Hp standards are commercially available making this assay the ideal candidate for transfer to the P-ELISA system. It is also a very relevant assay for investigation into future POC or "animal-side" diagnostics as the possibility of detecting the level of $\mathrm{Hp}$ at the POC, will allow for disease to be diagnosed in a timely and cost-effective manner, improving overall animal health and welfare [27].

This study describes a novel fabrication method for realisation of a hybrid paper-based microfluidic platform, termed multi-pad paper plate $\left(\mathrm{MP}^{3}\right)$, which can produce a device compatible with standard 96-well microplate format allowing rapid and low-cost translation and optimisation of laboratorybased ELISA assay into P-ELISA. The model used for the translation and optimisation of a laboratorybased assay using this platform is reported and is based on the development of a standard curve for detection of haptoglobin. The optimised protocol was further tested by analysing bovine serum samples with known $\mathrm{Hp}$ concentrations and by comparing results with the existing laboratory-based ELISA.

\section{Experimental}

\section{1. $\mathrm{MP}^{3}$ design and fabrication}

The $\mathrm{MP}^{3}$ was fabricated using a laser micromachining of paper and thermal lamination technique. $\mathrm{A}$ 3D mechanical computer-aided design (CAD) (SolidWorks 2012, USA) was used to design both the paper device and the plastic lamination used for device packaging (Fig. 1). Whatman Grade 1 (WHT 1) cellulose chromatography paper (Sigma-Aldrich, UK) and $75 \mu$ gloss laminating pouches (Staples, UK) were cut using a class $2 \mathrm{CO}_{2}$ laser (Speedy 300 Laser Engraver - Trotec). The optimised settings for 
cutting both materials were: power $4 \%$, speed $0.4, \mathrm{PPI} / \mathrm{Hz} 1000$, passes 1 , air assist on. These settings were chosen as the best combination for fast and precise production, while avoiding material burning. A range of settings were evaluated to achieve the high resolution required, while maintaining fast production (minimum time possible for fabrication) and ease of removal of devices (both paper and lamination sheets not requiring manual trimming or tearing). Increasing power settings to above $4 \%$ (i.e. $0.16 \mathrm{~W}$ ) was associated with burning (darkening) of the paper edges, while below the $4 \%$ threshold there was incomplete cutting of the material. When the speed was increased to 0.6, paper had to be manually detached from the main sheet, with high risk of tearing the microchannels $(0.5 \mathrm{~mm})$ and making the device not usable.

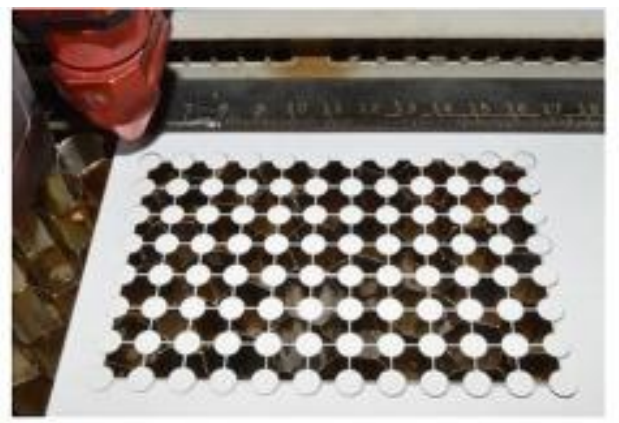

A)

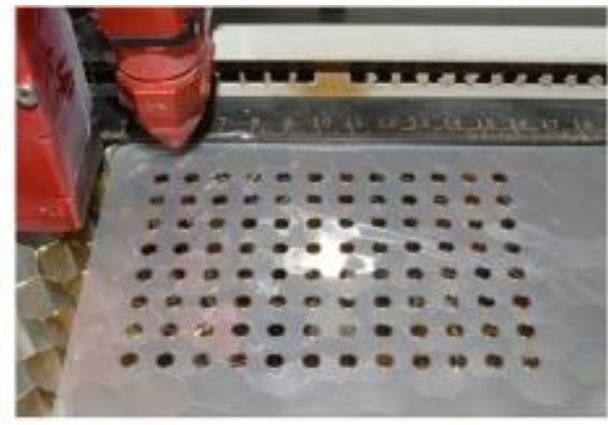

B)

\section{A)}

A)

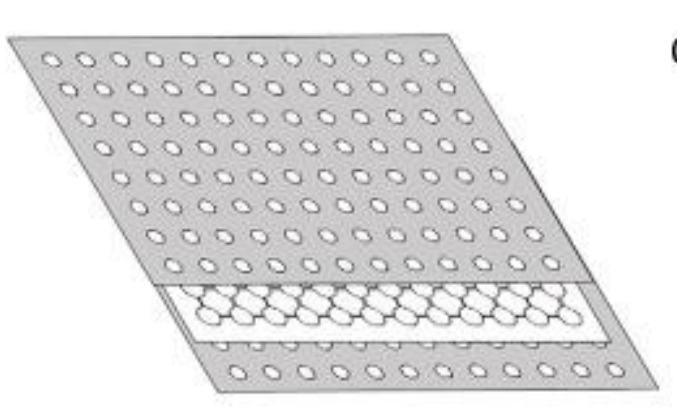

C)

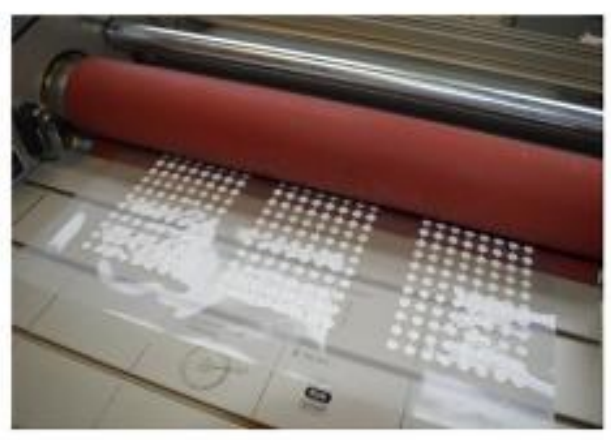

D)

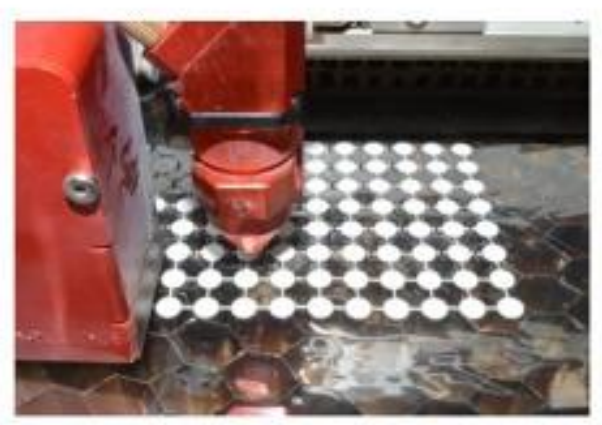

E)

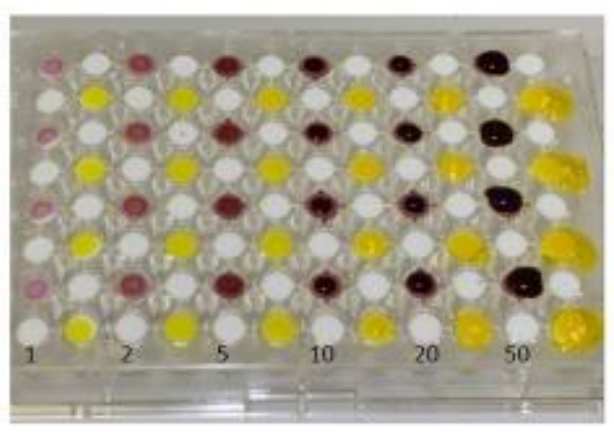

F)

Figure 1 Schematic of the fabrication of the MP3. A) Paper cut with a laser cutter. B) Laminating pouches cut with a laser cutter. C) Assembly of device in the pre-cut lamination sheets. D) Lamination of the device. E) Severing of interconnecting paper channels by laser cutter. F) Application of two different food colours (red and yellow) to the device with no evidence of cross-contamination between pads. Numbers represent volume of fluids applied $(\mu \mathrm{l})$.

Paper and lamination sheets were cut in the desired format and then laminated to produce the final device. The MP ${ }^{3}$ consists of 96 circular paper pads of $6 \mathrm{~mm}$ diameter connected by a channel of $0.5 \mathrm{~mm}$ width and $3 \mathrm{~mm}$ in length (Fig. 1A) and a complementary lamination sheet of 96 circular holes of $4 \mathrm{~mm}$ diameter (Fig. 1B), assembled in the pre-cut lamination sheet (Fig. 1C) and laminated at $110^{\circ} \mathrm{C}$ at the lowest speed setting (Fig. 1D) using a roll laminator (GBC Catena 35 Roll Laminator). After lamination, the laminated $\mathrm{MP}^{3}$ was placed back into the laser and the connecting microchannels between pads 
were severed by the laser cutter (Fig. 1E), making each pad completely independent from the others. During this final step of fabrication, plate information including numbers, letters and other information were cut or engraved on the final device. Optimisation was also carried out for this second cutting process. To keep the same power and speed settings already optimised, a second cut (passes 2) was necessary to completely severe the channels without requiring additional manual handling.

The final device was tested with food colour (Morrison Natural Food Colouring Blue) for determination of the optimum volume of liquid to allow complete wetting of each pad (Fig. 1F). Alterations to the CAD design by scaling down pad and channel dimensions as well as by introducing different areas for cutting, allowed for immediate variation of the $\mathrm{MP}^{3}$ platforms produced.

\subsection{Translation and optimisation of a lab-based assay into a P-ELISA}

Translation of the protocol was achieved by evaluating and comparing different variables for each of the assay parameters (Table 1 ) on the $\mathrm{MP}^{3}$. In order to obtain the best possible signal with the minimum volume and concentration of reagents, a suitable timing for the immunoreactions and a functional washing technique were optimised. Signal intensity was measured by scanning the $\mathrm{MP}^{3}$ using a desktop scanner (Perfection V350 photo, Epson, UK, set to "color photo scanning", 1200 dpi resolution) and images were transformed in 8-bit greyscale with PixBuilder Studio 2.2 (WnSoft Ltd., UK). Mean gray value (mean pixel intensity) was measured with Image J (1.48v, National Institute of Health, USA), after image inversion and after oval selection of the area to be measured. Variables which provided the strongest signal (highest pixel intensity) and the lowest background noise were selected.

Table 1 Assay parameters assessed to optimise the P-ELISA (in chronological order of evaluation). Text in bold represents the variables with the strongest signal (highest pixel intensity) and the lowest background noise.

\begin{tabular}{llll}
\hline Assay parameters & \multicolumn{3}{l}{ Variables } \\
\hline Conjugate antibody dilution & $1: 500$ & $1: 750$ & $1: 1000$ \\
Number of washes & 2 & 3 & 4 \\
Washing with absorbent pad underneath MP3 & Yes & No & \\
Washing vacuum pressure & $-10 \mathrm{~Pa}$ & $-30 \mathrm{~Pa}$ & $-50 \mathrm{~Pa}$ \\
Amount of washing & $20 \mu \mathrm{l}$ & $50 \mu \mathrm{l}$ & $100 \mu \mathrm{l}$ \\
Conjugate antibody incubation time & $60 \mathrm{~s}$ & $\mathbf{9 0 s}$ & $150 \mathrm{~s}$ \\
Hp incubation time & $5 \mathrm{~min}$ & $\mathbf{1 0 m i n}$ & $15 \mathrm{~min}$ \\
Capture antibody dilution & $1: 500$ & $\mathbf{1 : 1 0 0 0}$ & $1: 2000$ \\
Capture antibody incubation time & $5 \mathrm{~min}$ & $\mathbf{1 0 m i n}$ & $15 \mathrm{~min}$
\end{tabular}

\subsection{Sandwich Hp P-ELISA protocol}


Based on the translation and optimisation of the lab-based protocol, a sandwich P-ELISA for the detection of bovine $\mathrm{Hp}$ was applied to the $\mathrm{MP}^{3}$. Purified rabbit anti-bovine haptoglobin IgG (Life Diagnostics, USA; Catalogue No. 18120) was diluted to 1:1000 in wash buffer [WB (0.02 M Tris-HCl with $0.05 \%$ Tween-20, $\mathrm{pH}$ 7.4)]. A volume of $2 \mu \mathrm{l}$ of the prepared solution was deposited onto each pad and the paper allowed to dry at room temperature (RT) for $10 \mathrm{~min}$. Non-specific binding was limited by the addition of $2 \mu \mathrm{l}$ of blocking buffer [BB (10\% (w/v) Marvel milk protein in WB)] followed by air drying at RT for $10 \mathrm{~min}$. Standard bovine haptoglobin $(0.93 \mathrm{mg} / \mathrm{ml}$, Life Diagnostics Inc., USA) was diluted 1:5 in WB and then double diluted; $2 \mu$ of neat $\mathrm{Hp}$ and of each dilution were deposited onto the pads (sequentially in each column) and allowed to react for $10 \mathrm{~min}$ at RT. Plates were washed with $100 \mu \mathrm{l}$ WB by placing the plate in a 96 well plate vacuum manifold (Bethesda Research Laboratories, UK) connected to a vacuum pump (Millipore Chemical Duty Vacuum/pressure Pump, $115 \mathrm{~V} / 60 \mathrm{~Hz}, \mathrm{UK})$, with an absorbing pad made from Whatman Grade 1 cellulose chromatography paper (Sigma-Aldrich, UK) placed underneath the plate. After filling all wells of the manifold, the vacuum was activated $(-10 \mathrm{~Pa})$ and remained on until all wash buffer was cleared from the wells. This procedure was repeated three times. The purified rabbit anti-bovine haptoglobin IgG was conjugated with alkaline phosphatase (Lightning-Link ${ }^{\text {Tw }}$ Alkaline Phosphatase Conjugation Kit, Innova biosciences, Cambridge, UK; Catalogue No. 702-0010) according to the manufacturer's instructions then diluted 1:750 in WB. A volume of $4 \mu \mathrm{l}$ was then deposited onto each pad of the paper plate and incubated at RT for $90 \mathrm{~s}$. Plates were washed as described above. A volume of $4 \mu \mathrm{l}$ of ready-to-use buffered alkaline phosphatase substrate solution (BCIP®/NBT Blue Liquid Substrate System for Membranes by SigmaAldrich (Catalogue No B3804)) was added to each pad. After $10 \mathrm{~min}$ at RT, the paper plates were scanned and analysed as described in 2.2.

To evaluate the performance of the $\mathrm{MP}^{3}$ with biological samples, bovine serum samples with known levels of $\mathrm{Hp}$ were also analysed using the $\mathrm{MP}^{3}$ and the optimised sandwich $\mathrm{Hp}$ ELISA protocol (Fig. 2) and compared with results obtained from the same samples by a standard lab-based ELISA. A total of 8 blood samples, sourced from clinically healthy lactating cattle from a commercial dairy farm but showing a variable range of circulating $\mathrm{Hp}$, were analysed at a 1:10 dilution in $\mathrm{WB}$, in triplicate on two separate $\mathrm{MP}^{3}$ devices.

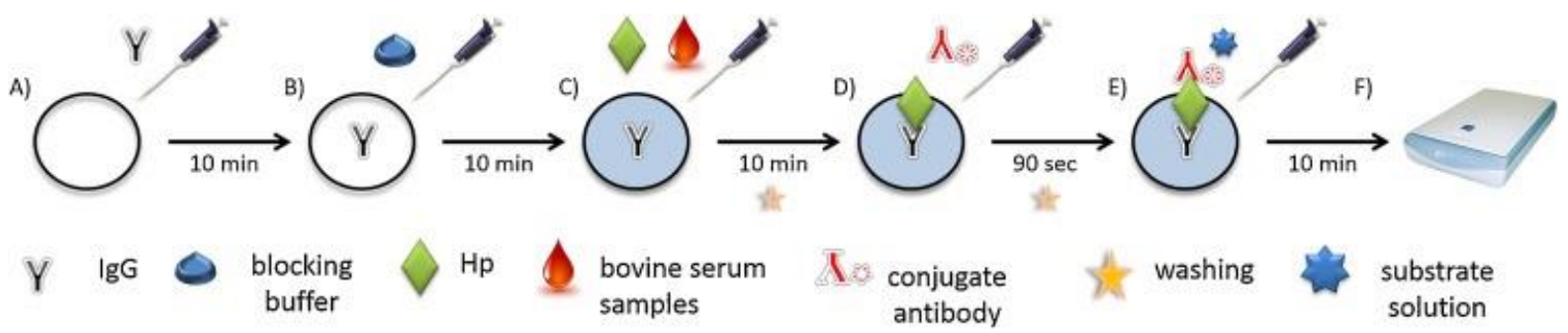

Figure 2 Schematic representation of the sandwich Hp P-ELISA for the detection of Hp in bovine serum as applied to the MP3. A) Deposition of $2 \mu \mathrm{l}$ rabbit anti-bovine IgG diluted 1:1000 in WB. B) Blocking with $2 \mu$ l BB. C) Deposition of $2 \mu$ l standard Hp diluted 1:20 then double diluted to 1:1280 and $2 \mu$ l bovine serum sample diluted 1:10 D) After washing, addition of $4 \mu l$ conjugate diluted 1:750 in WB. E) After washing, addition of $4 \mu$ l substrate. F) MP3 scanned using a flatbed desktop scanner. All steps of the assay were performed at room temperature.

\section{Results}

3.1. $\mathrm{MP}^{3}$ production and application 
Following the method described, it was possible to produce a platform with 96 completely independent circular pads, which was compatible with instruments commonly used in laboratories for 96 well microplates (Fig. 3A). There was no evidence of leakage between pads, even when excess fluid $(50 \mu \mathrm{L})$ was deposited. A volume of $2 \mu \mathrm{l}$ was determined as the ideal amount to obtain complete wetting of the pads whilst minimising the drying time required at room temperature. The total time to produce one A4 sheet containing 3 devices was under $10 \mathrm{~min}$, with a resolution of fabrication technology to $0.5 \mathrm{~mm}$ channels (Fig. 3B). Furthermore, precise laser cutting of $3.5 * 0.5 \mathrm{~mm}$ square holes between interconnected channels was achieved, by calculated alignment of the laser pointer with the produced device. This second laser cutting process also allowed for pre-designed numbers and characters to be included in the final device (Fig. 3B). The thickness of each device was $0.3 \mathrm{~mm}$, with up to $50 \mathrm{MP}^{3}$ occupying the same space as a standard $19 \mathrm{~mm}$ height 96-well microplate.

A)

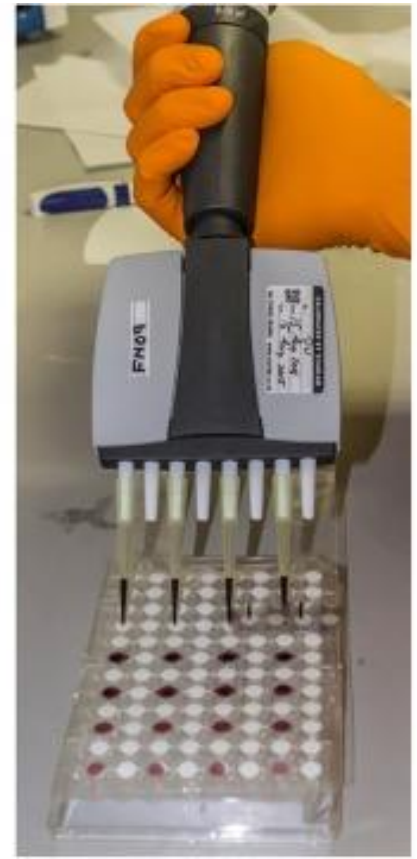

B)

D)

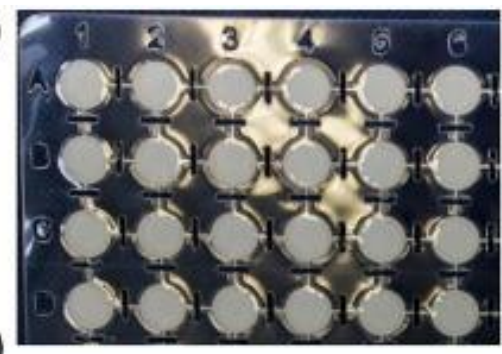

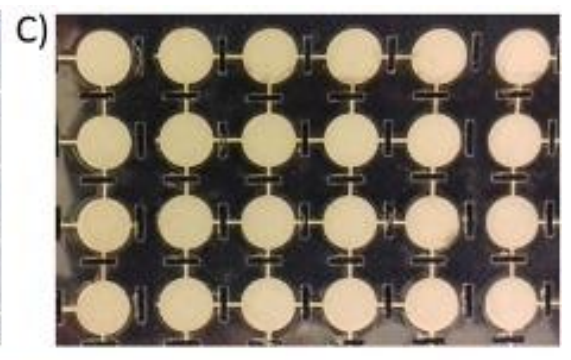

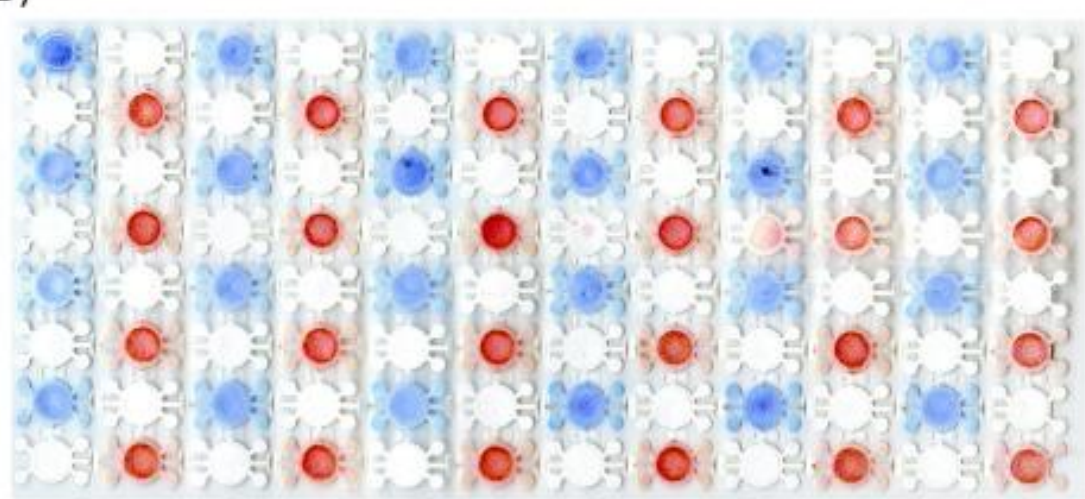

Figure $3 \mathrm{MP}^{3}$ applications and production techniques. A) Colour application on MP3 using multichannel pipette. B) $M P^{3}$ showing precise paper cutting of channels and final device engraving with letters and numbers. C) $M P^{3}$ showing removal of the interconnecting microchannels at every $4^{\text {th }}$ column to include 3 different types of paper on the same device. D) Modified $M P^{3}$ with 96 central pads of $3 \mathrm{~mm}$ radius each connected to 4 smaller test zones of 2 mm radius and connecting channels of $1 \mathrm{~mm}$. Application of two different food colours (10 $\mu /$ red and blue food colour dye) showed each test zone to be independent with solution expanding to the entire test zone, without cross-contamination of the adjacent areas.

Through the alterations of the CAD design, it was also possible to produce two modified versions of the $\mathrm{MP}^{3}$. By removing the interconnecting microchannels at every 4th column (Fig. $3 \mathrm{C}$ ) an alternative version allowed the inclusion of three different paper types in a single device (providing 12 independent pads per paper type). This allowed WHT 1, Whatman filter paper Grade 4 (pore size 20$25 \mu \mathrm{m}$ ) and Whatman filter paper Grade 597 (pore size 4-7 $\mu \mathrm{m}$ ) to be included on the same device. A different version of the $\mathrm{MP}^{3}$, comprising 480 pads, was also produced by having each of the 96 central pads connected with 4 microchannels ( $1 \mathrm{~mm} \times 0.72 \mathrm{~mm}$ ) to 4 smaller pads ( $2 \mathrm{~mm}$ diameter), to allow for multiple tests to be run at the same time on the same sample (4 tests/sample) (Fig. 3D).

\subsection{Sandwich Hp P-ELISA}

Using the $\mathrm{MP}^{3}$, a sandwich Hp P-ELISA was successfully performed, demonstrating that increasing concentrations of bovine standard $\mathrm{Hp}$ resulted in increasing intensity responses, based on a 
colorimetric reaction and visible with the naked eye (Fig. 4A). Average pixel intensity values for each circular pad were analyzed in GraphPad Prism 5 (GraphPad Software, Inc., USA) and correlated with the different concentrations of $\mathrm{Hp}$. A standard curve was generated using non-linear regression and a sigmoid curve response (variable slope) was obtained (Fig. 4B), showing high correlation between pixel intensity and $\mathrm{Hp}$ concentration $\left(R^{2}=0.85\right)$. The limit of detection (LOD) achieved was $0.73 \mu \mathrm{g} / \mathrm{ml}$, calculated as three times the standard deviation of the negative control sample. Plateau of the signal intensity was found at an $\mathrm{Hp}$ concentration of $23.25 \mu \mathrm{g} / \mathrm{ml}$.

A)

B)
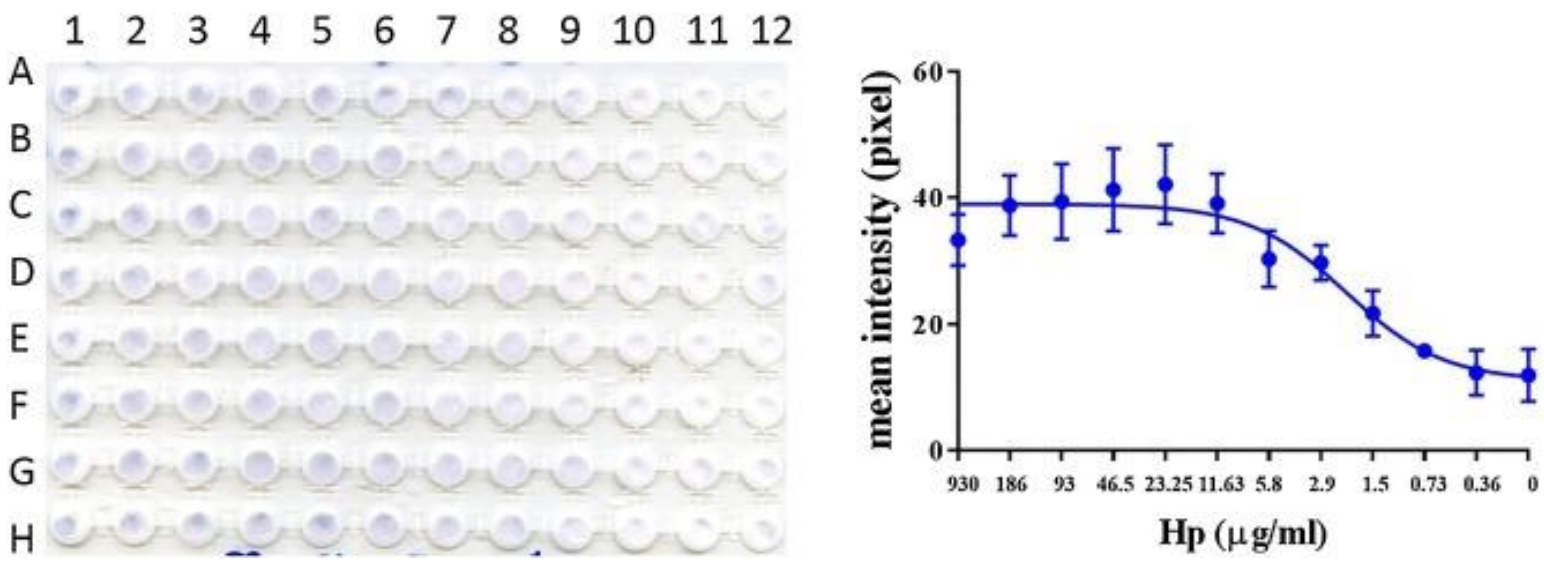

Figure 4 Sandwich Hp ELISA on MP3. A) Column 1-11 double dilution of $\mathrm{Hp}(1=930 \mu \mathrm{g} / \mathrm{ml}$ to $11=0.36 \mu \mathrm{g} / \mathrm{ml})$ and 12 negative control $(W B)$. Rows A to H replicates $(n=8)$. B) Non-linear regression (variable slope) of mean pixel intensity in relation to $\mathrm{Hp}$ concentration $(\mu \mathrm{g} / \mathrm{ml})$. Each data point represents the mean of 8 replicate of $\mathrm{Hp}$ measurements, shown as 10 logarithmic conversion of $\mathrm{Hp}$ concentration. Error bars represent standard deviation. Linear range $0.73-23.25 \mu \mathrm{g} / \mathrm{ml}$.

The levels of $\mathrm{Hp}$ in clinical blood samples were also assessed with the optimised P-ELISA on the $\mathrm{MP}^{3}$ and compared using Pearson correlation to those obtained from the standard lab-based ELISA protocol. There was a significant correlation $(p<0.05)$ between the average measurements obtained from the lab-based ELISA and the MP ${ }^{3}$ P-ELISA. Hp values $(\mu \mathrm{g} / \mathrm{ml})$ calculated from the average OD measurements from each sample were also compared using a paired t-test, showing that there was no difference $(p=0.75)$ between values obtained with the lab-based ELISA and the P-ELISA performed on the $\mathrm{MP}^{3}$ (Fig. 5). 


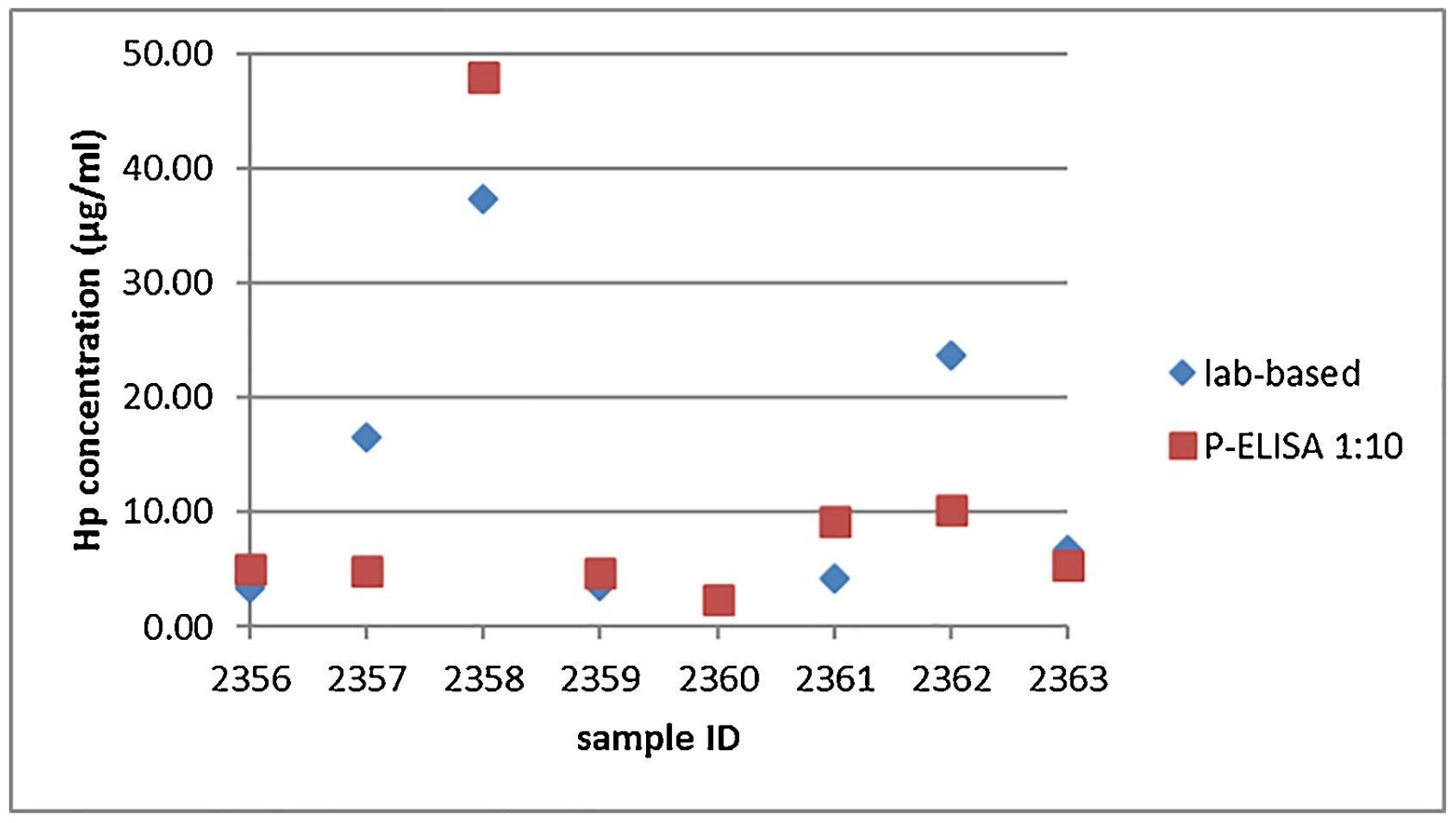

Figure 5 Levels of $\mathrm{Hp}(\mu \mathrm{g} / \mathrm{ml})$ in clinical blood samples calculated from the results obtain by the lab-based assay and the $P$ ELISA on the MP3.

\subsection{Cost-benefit analysis of $M P^{3}$ sandwich Hp P-ELISA}

The total time required to perform a complete P-ELISA on the $\mathrm{MP}^{3}$ was just over 40 min and multiple assays (up to 3 ) could be processed at the same time. The time and cost for performing the sandwich Hp P-ELISA was considerably reduced when compared to the same assay performed in the traditional lab-based format, where reductions of $88 \%$ in cost and $93 \%$ in time where achieved (Table 2). A cost per sample of $£ 0.13$ (when samples were run in duplicate on each device) was calculated versus a cost of $£ 1$ per sample for the standard lab-based ELISA.

Table 2 Cost-benefit analysis of performing the sandwich Hp ELISA on the MP3 or with the lab-based technique. \# negligible cost.

Hp P-ELISA on MP3

Lab-based Hp ELISA

\begin{tabular}{|c|c|c|c|c|c|c|}
\hline & $\begin{array}{l}\text { Volume } \\
(\mu \mathrm{L})\end{array}$ & $\begin{array}{l}\text { Time } \\
(\min )\end{array}$ & $\begin{array}{l}\text { Cost per } \\
\text { plate }\end{array}$ & $\begin{array}{l}\text { Volume } \\
(\mu \mathrm{L})\end{array}$ & $\begin{array}{l}\text { Time } \\
(\min )\end{array}$ & $\begin{array}{l}\text { Cost per } \\
\text { plate }\end{array}$ \\
\hline Primary antibody & 2 & 10 & f1 & 100 & 480 & f5.5 \\
\hline Blocking buffer & 2 & 10 & $\#$ & 200 & 60 & $\#$ \\
\hline Antigen (Hp) & 2 & 10 & $f 1.2$ & 100 & 60 & f15 \\
\hline $\begin{array}{l}\text { Conjugated } \\
\text { antibody }\end{array}$ & 4 & 1.5 & f3.4 & 100 & 60 & $£ 20$ \\
\hline Substrate & 4 & 10 & $£ 0.4$ & 100 & 10 & f10.5 \\
\hline Plate & - & - & $f 0.3$ & - & - & f1.5 \\
\hline Total & 14 & 41.5 & f6.3 & 600 & 670 & $f 52.5$ \\
\hline
\end{tabular}


To translate and optimise the lab-based ELISA onto P-ELISA a total of $62 \mathrm{MP}^{3}$ where used, which represent a cost of translation just below $£ 400$. To obtain the standard curve a total of $6 \mathrm{MP}^{3}$ where used and a further $10 \mathrm{MP}^{3}$ where used to apply the $\mathrm{Hp}$ with serum.

\section{Discussion}

Here we describe a novel fabrication method to create a hybrid paper-based microfluidic device. The device generated, termed multi-pad paper plate $\left(\mathrm{MP}^{3}\right)$ has proven to be low-cost, rapid and simple to produce and can be used in serological testing via ELISA. Some of the main limitations of other techniques available (e.g. cumbersome production and low-resolution technique) have been resolved, whilst offering a very robust platform (which can sustain vacuum pressure, high volumes of fluid and high temperatures) and a very flexible fabrication method (with the possibility of engraving/cutting of desired information on the device, incorporation of different materials and modification of the initial design for multiplex analysis). A laser cutter and a laminator are required, but no other specialised machinery or clean room facility are needed. Class 2 laser cutters are easily available within Universities and are often employed for other paper device fabrication methods, where masks or other devices are usually needed $[4,28,29]$. Computer-controlled knife plotters have also been used for production of paper-based devices by paper cutting, but three sequential overlapping cuts where required to precisely cut the chromatography paper without tearing [30]. Although a professional laser machine was employed in this case, commercially available $\mathrm{CO}_{2}$ laser cuttingmachines could potentially be used, which have a similar price to solid-ink printers, commonly used for wax printing of paper-based devices. Furthermore, the wax printing technique lacks the high resolution achieved by other methods [7], due to the wax spreading by capillary action [31]. In this case, dimensions are the same from design to production of the final device and a resolution of $500 \mu \mathrm{m}$ (Fig. 3B) could be achieved. Although precision may not be the priority for P-ELISA platforms, it is the case for more complicated designs, like 3D paper-based devices. Moreover, wax is soluble in organic compounds, which would have made this platform unsuitable for assays in which reagents are solubilised in urea [32]. This method also eliminates the need for chemical treatments, which can pose health and safety issues and increase the overall costs of fabrication. Furthermore, lamination affords extra strength to the device, making it especially robust but still manageable. At the same time, correct assembly techniques can sometimes be an issue with these devices, therefore the use of a matrix of interconnecting microchannels between pads, a complete novel approach in the fabrication of these devices, allowed for easy and rapid device assembly, minimising inter batch differences and reducing wastage.

Another considerable advantage of this system, compared to other methods, is the high degree of flexibility when designing a new device. Different prototypes can be created from easily made alterations to the original CAD file, without requiring purpose-built devices (e.g. masks), which allows for different conditions and/or parameters to be tested in a cost and time efficient manner. Paper of different pore sizes could be assembled on the same device, allowing optimisation of performance factors such as flow rate and reaction time or for developing immunoassays that require different conditions to be carried out at the same time, with the possibility of enhancing functionality. It also gives the possibility of using different materials on the same device, as other materials have sparked much interest in the field of low-cost POC devices [16]. Others have already shown the advantages in combining paper and cotton to reduce cross-contamination of samples [16] and the recently characterised class of hybrid paper microfluidic platforms show that paper could solve some of the 
intrinsic limitations of other substrates [17]. Combining different materials to enhance the performance of microfluidic platforms is showing favourable results in improving operator control over specific assay variables [33]. Due to the superior strength of the device provided by the lamination, it was possible to apply vigorous washing through a vacuum manifold (up to $-50 \mathrm{~Pa}$ ), which reduced washing time and allowed greater control over other variables, as there is less operator variability between plates than by standard pipette washing and removal of wash buffer through tapping out onto absorbent paper towels. High volumes of fluid (up to $100 \mu \mathrm{l}$ ) could also be applied to the device without cross-contamination or damage.

As the spatial distances between the circular pads were maintained, as in the traditional plastic 96well microplate format, common instruments used in the lab (e.g. multichannel pipettes and washing manifolds) can also be used with the new format. This allows the platform to be used for translation and optimisation of a laboratory-based ELISA into P-ELISA, using a model assay for determination of bovine $\mathrm{Hp}$ in serum. The results showed that it was possible to transfer the assay onto the paper format with considerable reductions in both assay time and cost. The colorimetric reaction generated on paper demonstrated that the colour intensity was proportional to the dilution of $\mathrm{Hp}$, with semiquantitative detection being possible using an $\mathrm{Hp}$ calibration curve. The colour intensity was analysed using a desktop scanner, however a future development could incorporate the use of a mobile smartphone camera to reduce reliance on bulky machinery which would not suit the definition of POC [34]. It could also involve the use of an app to assist in analysis and interpretation of the test outcome at the animal side, with the possibility for data storage. The linear range achieved was $0.73-$ $23.25 \mathrm{\mu g} / \mathrm{ml}$ and based on the expected level of $\mathrm{Hp}$ below $0.02 \mathrm{mg} / \mathrm{ml}$ for healthy cows [24], a sample dilution of 100 -fold would be below the LOD for clinically healthy animals. Cases of mastitis have shown to be above $0.74 \mathrm{mg} / \mathrm{ml}$ [24], which at the 100 -fold dilution would fall within the detectable range. The $\mathrm{MP}^{3}$ was able to detect different levels of $\mathrm{Hp}$ in biological samples (serum), with significant correlation between results obtained from a lab ELISA and the same ELISA performed on the MP ${ }^{3}$. Of the 8 samples analysed, 6 showed comparable results between the two assays, while two (sample 2357 and 2362) had lower signal than the lab-format results. Further work is currently being undertaken to compare the values obtained using the lab-assay and the P-ELISA for evaluation within clinical settings. While the LOD achieved was 100 -fold above that achievable in a commercial labbased ELISA $\left(7.8 \mathrm{ng} / \mathrm{ml}^{1}\right)$ this may not necessarily represent a constraint to its use in veterinary diagnostics, where emphasis is placed more on the achievement of cost-effective, high throughput and rapid testing. While the present format cannot be considered as truly POC, the possibility remains to use it within veterinary clinics or within remote labs, as timing is considerably reduced and the materials can be sold in a kit format (with packaging of $50 \mathrm{MP}^{3}$ in the space occupied by a single standard 96-well microplate). Furthermore, biomarkers of disease such as acute phase proteins usually need to be coupled with other specific disease tests or require other APPs to accurately determine disease status [35]. Therefore, using a modification of this platform (Fig. 3D), other biomarkers (up to 4/sample) of veterinary interest could be translated onto P-ELISA, in a multiplex format, making the transition from the lab-based to the paper format both time-efficient and costeffective and allowing for multiple tests to be run in parallel.

This technique also offers the possibility of having built-in information, without the need for changes to the original design (Fig. 3B). A custom-made device could be built, where specific information (e.g. farm holding/animal number or disease to be tested) are added, upon request, to the basic platform. This could minimize end-user input (information the operator must add manually) and operator error (no need for hand writing of information). Furthermore, due to the high melting point of laminated sheets (devices can sustain laminating temperatures of $110^{\circ} \mathrm{C}$ ), the use of this platform would be 
suitable in high temperature settings (e.g. no restriction on the storage of the device at tropical/subtropical ambient temperature).

Although the technology is, at the moment, suitable specifically for laboratory prototyping, future high-throughput industrial manufacturing could be envisaged. A digital laser cutter and laminating machine, like those used in the label making industry, could be employed. Combining the two fabrication processes in a single machine would decrease the total production time, as well as manual operations to a minimum. More devices per sheets could also be produce by using larger paper sheets (i.e. A0). In the context of low-resource settings, a capture-antibody pre-loaded stack of devices with clear instructions and reference charts for colour comparison could be prepared and sent to remote labs for on-site testing by untrained personnel. The platform could also be used to translate other assays into the paper format, following the same systematic approach presented here and therefore increasing the scope of diagnostic tests available.

\section{Conclusions}

In conclusion, the fabrication technique developed here is a valid alternative to produce platforms used to perform P-ELISA previously described. The new method of production is simple, rapid and of high-resolution and can produce robust, versatile and low-cost devices, providing 96 completely independent paper pads and compatible with standard laboratory microplates and instruments. Furthermore, there was a reduction of $93 \%$ in time and of $88 \%$ in cost of performing a diagnostic assay. The method is well suited for accessible fabrication of lab prototyping devices for rapid optimisation of assay conditions, which could potentially be used to transfer any type of existing ELISA into P-ELISA. Additionally, the manufacturing method is also scalable for high volume manufacturing, producing large quantities of paper based microfluidic devices for POC applications as well as particularly suitable for low-resource settings. While it cannot be considered a POC device at this stage, it still offers the opportunity to carry out testing "in-house" (e.g. within veterinary clinics) due to the considerably reduced assay time, reagent costs and reliance on limited infrastructure. Further studies will focus on the evaluation of the analytical performance of the Hp P-ELISA, toward its use as diagnostic test in clinical settings, as well as looking at exploiting this new fabrication technique to develop a 3D MPAD to enable true POC testing at the "animal-side".

\section{Acknowledgements}

The authors would like to thank Moredun Scientific Ltd. and Heriot-Watt University for funding this research and Tom McNeilly, Mairi Mitchell and David Eckersall for the support with the Hp assay. We would also like to thank Alan Faulkner-Jones for the help and support with the laser cutting and image analysis.

\section{References}

[1] K.N. Han, C.A. Li, G.H. Seong, Microfluidic chips for immunoassays, Annu. Rev. Anal. Chem. 6 (6) (2013) 119-141.

[2] W.A. Zhao, A. van den Berg, Lab on paper, Lab Chip 8 (2008) 1988-1991. 
[3] A.W. Martinez, Microfluidic paper-based analytical devices: from POCKET to paperbased ELISA, Bioanalysis 3 (2011) 2589-2592.

[4] E. Carrilho, S.T. Phillips, S.J. Vella, A.W. Martinez, G.M. Whitesides, Paper microzone plates, Anal. Chem. 81 (2009) 5990-5998.

[5] A.C. Glavan, D.C. Christodouleas, B. Mosadegh, H.D. Yu, B.S. Smith, J. Lessing, et al., Folding analytical devices for electrochemical ELISA in hydrophobic RH paper, Anal. Chem. 86 (2014) 1199912007.

[6] C.-K. Hsu, H.-Y. Huang, W.-R. Chen, W. Nishie, H. Ujiie, K. Natsuga, et al., Paperbased ELISA for the detection of autoimmune antibodies in body fluid-The case of bullous pemphigoid, Anal. Chem. 86 (2014) 4605-4610.

[7] R.C. Murdock, L. Shen, D.K. Griffin, N. Kelley-Loughnane, I. Papautsky, J.A. Hagen, Optimization of a paper-based ELISA for a human performance biomarker, Anal. Chem. 85 (2013) 11634-11642.

[8] S. Wang, L. Ge, X. Song, J. Yu, S. Ge, J. Huang, et al., Paper-based chemiluminescence ELISA: lab-onpaper based on chitosan modified paper device and waxscreen- printing, Biosens. Bioelectron. 31 (2012) 212-218.

[9] A. Apilux, Y. Ukita, M. Chikae, O. Chailapakul, Y. Takamura, Development of automated paperbased devices for sequential multistep sandwich enzyme-linked immunosorbent assays using inkjet printing, Lab Chip 13 (2013) 126-135.

[10] C.M. Cheng, A.W. Martinez, J. Gong, C.R. Mace, S.T. Phillips, E. Carrilho, et al., Paper-based ELISA, Angew. Chem. 49 (2010) 4771-4774 (International ed in English).

[11] L. Ge, S. Wang, X. Song, S. Ge, J. Yu, 3D origami-based multifunction-integrated immunodevice: low-cost and multiplexed sandwich chemiluminescence immunoassay on microfluidic paper-based analytical device, Lab Chip 12 (2012) 3150-3158.

[12] D.R. Ballerini, X. Li, W. Shen, Patterned paper and alternative materials as substrates for low-cost microfluidic diagnostics, Microfluid Nanofluid 13 (2012) 769-787.

[13] M. Dou, S.T. Sanjay, M. Benhabib, F. Xu, X. Li, Low-cost bioanalysis on paper-based and its hybrid microfluidic platforms, Talanta 145 (2015) 43-54.

[14] S.T. Sanjay, M. Dou, J. Sun, X. Li, A paper/polymer hybrid microfluidic microplate for rapid quantitative detection of multiple disease biomarkers, Sci. Rep. 6 (2016) 30474.

[15] M. Dou, D.C. Dominguez, X. Li, J. Sanchez, G. Scott, A versatile PDMS/paper hybrid microfluidic platform for sensitive infectious disease diagnosis, Anal. Chem. 86 (2014) 7978-7986.

[16] S.C. Lin, M.Y. Hsu, C.M. Kuan, H.K. Wang, C.L. Chang, F.G. Tseng, et al., Cottonbased diagnostic devices, Sci. Rep. 4 (2014) 6976-6988.

[17] P. Zuo, X. Li, D.C. Dominguez, B.-C. Ye, A PDMS/paper/glass hybrid microfluidic biochip integrated with aptamer-functionalized graphene oxide nano-biosensors for one-step multiplexed pathogen detection, Lab Chip 13 (2013) 3921-3928.

[18] P.D. Eckersall, F.P. Lawson, L. Bence, M.M. Waterston, T.L. Lang, W. Donachie, et al., Acute phase protein response in an experimental model of ovine caseous lymphadenitis, BMC Vet. Res. 3 (2007) 35-41. 
[19] P.M.H. Heegaard, D.L. Godson, M.J.M. Toussaint, K. Tjornehoj, L.E. Larsen, B. Viuff, et al., The acute phase response of haptoglobin and serum amyloid a (SAA) in cattle undergoing experimental infection with bovine respiratory syncytial virus, Vet. Immunol. Immunopathol. 77 (2000) 151-159.

[20] B.P. Holland, D.L. Step, L.O. Burciaga-Robles, R.W. Fulton, A.W. Confer, T.K. Rose, et al., Effectiveness of sorting calves with high risk of developing bovine respiratory disease on the basis of serum haptoglobin concentration at the time of arrival at a feedlot, Am J. Vet. Res. 72 (2011) 13491360.

[21] D.L. Godson, M. Campos, S.K. AttahPoku, M.J. Redmond, D.M. Cordeiro, M.S. Sethi, et al., Serum haptoglobin as an indicator of the acute phase response in bovine respiratory disease, Vet. Immunol. Immunopathol. 51 (1996) 277-292.

[22] B. Wells, G.T. Innocent, P.D. Eckersall, E. McCulloch, A.J. Nisbet, S.T.G. Burgess, Two major ruminant acute phase proteins, haptoglobin and serum amyloid a, as serum biomarkers during active sheep scab infestation, Vet. Res. 44 (2013) 103-114.

[23] H. Murata, N. Shimada, M. Yoshioka, Current research on acute phase proteins in veterinary diagnosis: an overview, Vet. J. 168 (2004) 28-40.

[24] P.D. Eckersall, F.J. Young, C. McComb, C.J. Hogarth, S. Safi, A. Weber, et al., Acute phase proteins in serum and milk from dairy cows with clinical mastitis, Vet. Rec. 148 (2001) 35-+..

[25] P.D. Eckersall, R. Bell, Acute phase proteins: biomarkers of infection and inflammation in veterinary medicine, Vet. J. 185 (2010) 23-27.

[26] R.F. Cooke, J.D. Arthington, Concentrations of haptoglobin in bovine plasma determined by ELISA or a colorimetric method based on peroxidase activity, J. Anim. Physiol. Anim. Nutr. (Berl.) 97 (2013) 531-536.

[27] V. Busin, B. Wells, M. Kersaudy-Kerhoas, W. Shu, S.T.G. Burgess, Opportunities and challenges for the application of microfluidic technologies in point-of-care veterinary diagnostics, Mol. Cell. Probes 30 (2016) 331-341.

[28] K. Abe, K. Kotera, K. Suzuki, D. Citterio, Inkjet-printed paperfluidic immuno-chemical sensing device, Anal. Bioanal. Chem. 398 (2010) 885-893.

[29] W. Dungchai, O. Chailapakul, C.S. Henry, A low-cost, simple, and rapid fabrication method for paper-based microfluidics using wax screen-printing, Analyst 136 (2011) 77-82.

[30] E.M. Fenton, M.R. Mascarenas, G.P. Lopez, S.S. Sibbett, Multiplex lateral-flow test strips fabricated by two-dimensional shaping, ACS Appl. Mater. Interfaces 1 (2009) 124-129.

[31] E. Carrilho, A.W. Martinez, G.M. Whitesides, Understanding wax printing: a simple micropatterning process for paper-based microfluidics, Anal. Chem. 81 (2009) 7091-7095.

[32] F.G. Nunn, S.T. Burgess, G. Innocent, A.J. Nisbet, P. Bates, J.F. Huntley, Development of a serodiagnostic test for sheep scab using recombinant protein Pso o 2, Mol. Cell. Probes 25 (2011) 212218.

[33] M.J. Uddin, G.J. Jin, J.S. Shim, A paper-plastic hybrid microfluidic device for smartphone based colorimetric analysis of urine, Anal. Chem. 89 (2017) 13160-13166. 
[34] A.W. Martinez, S.T. Phillips, E. Carrilho, S.W. Thomas, H. Sindi, G.M. Whitesides, Simple telemedicine for developing regions: camera phones and paper-based microfluidic devices for realtime, off-site diagnosis, Anal. Chem. 80 (2008) 3699-3707.

[35] P.D. Eckersall, Recent advances and future prospects for the use of acute phase proteins as markers of disease in animals, Revue De Medecine Veterinaire 151 (2000) 577-584. 